\title{
Beware the anterior lying recurrent laryngeal nerve in a retro-oesophageal goitre
}

A 36-year-old woman presented with a 4-month history of right neck lump. She had mild shortness of breath, but no dysphagia or voice changes. Clinical examination found a firm right thyroid mass that moved with swallowing, consistent with a right thyroid lesion. Ultrasound evaluation of the lump revealed a large lobulated isoechoic mass $(8.1 \times 5.4 \times 4.3 \mathrm{~cm})$ with cystic areas within the right thyroid lobe. Significantly, there was also a lobulated mass $(6.1 \times 5.1 \times 2.9 \mathrm{~cm})$ with similar sonographic features located posterior to the left thyroid lobe and oesophagus, which could have represented retro-tracheal extension from the right thyroid mass. Computed tomography scan revealed a large lobulated mass occupying most of the right thyroid lobe with cystic spaces. The mass extended posteriorly, displacing and compressing the oesophagus as well as the trachea with resultant significant luminal narrowing (Fig. 1). Fine-needle aspiration cytology of the right thyroid lobe showed features compatible with adenomatous goitre (Bethesda category II).

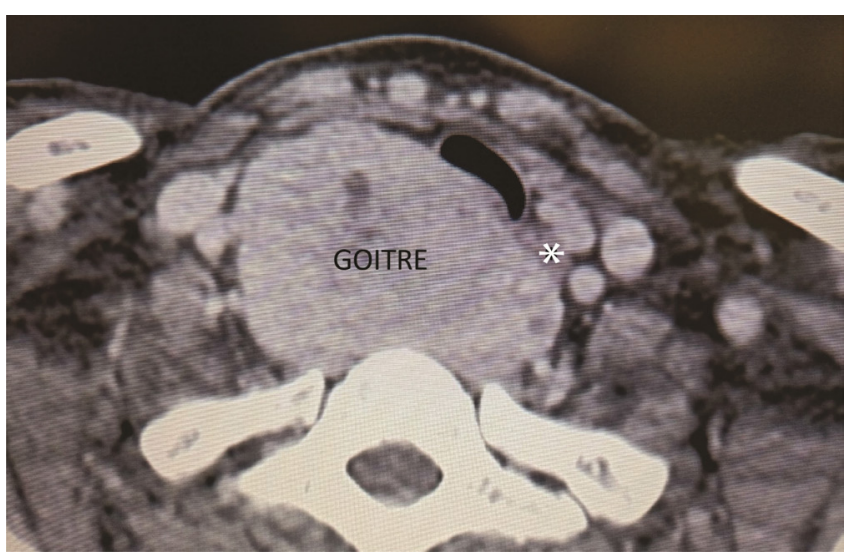

Fig. 1. Right thyroid goitre going retro-oesophageal (asterisk marks the oesophagus)

She underwent a right hemithyroidectomy. During the right hemithyroidectomy, where would you expect the right recurrent laryngeal nerve to be most commonly located?
A. Posterior and medial to the thyroid lobe
B. Superficial and lateral to the thyroid lobe
C. Anterior and lateral over the tubercle of Zuckerkandl
D. Medial to the inferior parathyroid gland
E. Lateral to the superior parathyroid gland

Discussion. The recurrent laryngeal nerve (RLN) is normally located posterior and medial to the thyroid lobe. ${ }^{1}$ The incidence of a superficial, lateral course of the RLN has been reported as $6-7 \%$ in primary thyroidectomy cases. This is always in associated with a tubercle of Zuckerkandl, where the nerve passes anterolaterally over the tubercle. ${ }^{2,3}$ Anterior lying nerves have also been reported in redo cases. ${ }^{2}$

In our patient, intraoperatively, there was a large right multinodular thyroid gland, which was retrooesophageal and retrosternal. Here we describe and show photographic evidence of a recurrent nerve that runs directly anteriorly, over the direct ventral surface of the thyroid lobe (Fig. 2). The occurrence of this in a previously unoperated patient is exceedingly rare, and to our knowledge there are no cases with photographic evidence reported in the literature.

On the right side of the neck, the nerve travels lateral to medial as it ascends at an angle towards the tracheaoesophageal groove and then parallel to it. As such, there

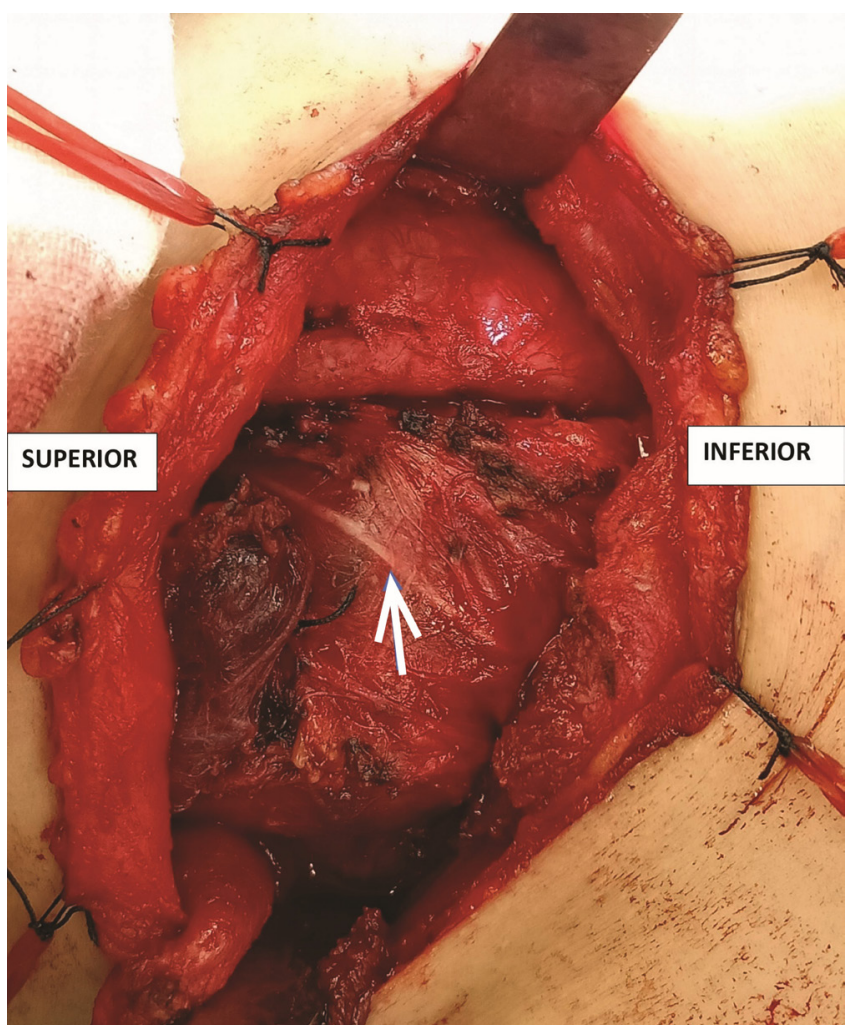

Fig. 2. Intraoperative photo showing a nerve (arrow) running anterior to the right thyroid goitre. (Colour figure available online.) 
is a potential space (both superficial and deep to the RLN) especially in the inferior central compartment. In our case, a retrograde posterior growth of the lobe that extended deep onto the nerve displaced the nerve to a more ventral location.

This case highlights that in a large thyroid nodule that is extending posteriorly (in this case retrooesophageal as well), the surgeon has to consider the unlikely but possible situation of the nerve being anterior to the gland.

Careful identification and dissection allowed preservation of the recurrent laryngeal nerve (Fig. 3) Postoperatively, the patient reported a normal voice. Both vocal cords were mobile on postoperative nasoendoscopy.

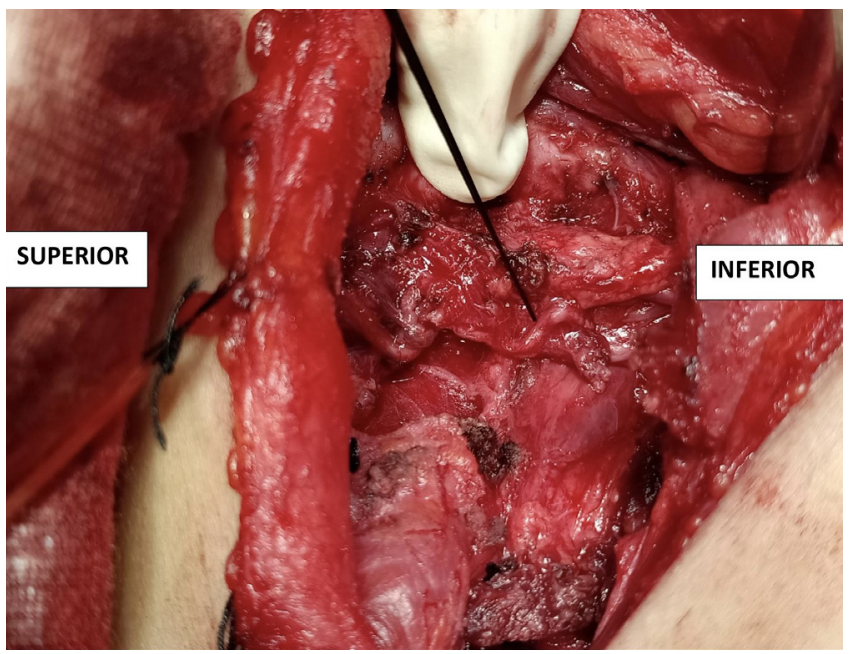

Fig. 3. Recurrent laryngeal nerve post resection. (Colour figure available online.)

In this case, the superficial and anterior lying position of the nerve was not obvious. There was a layer of fascia over the nerve that obscured the nerve visually. However, in anticipation of the neve being pushed to an unusual position, the surgeon with the aid of an intermittent nerve monitor found the nerve running directly anterior to the gland.

In general, the recurrent laryngeal nerve may be found in a more unusual location in a large multinodular goitre, and safe identification of the nerve can be achieved by finding it in a location where it is more consistent, such as the cricothyroid joint. The nerve can also be better preserved if one performs a capsular dissection, keeping very close to the gland to avoid injury to the nerve. ${ }^{4}$

To our knowledge, this is one of the first cases that demonstrates photographic evidence of a purely ventral lying nerve in a previously unoperated case in the literature. This case clearly highlights the specific configuration of a very large thyroid nodule that is growing posteriorly, especially a retro-oesophageal nodule, in which the surgeon has to be aware that the nerve may be in an unexpected position. Intermittent nerve monitoring may be a useful adjunct in this scenario. ${ }^{5}$

\section{REFERENCES}

1. Fancy T, Gallagher D 3rd, Hornig JD. Surgical anatomy of the thyroid and parathyroid glands. Otolaryngol Clin North Am 2010;43:221-7, vii.

2. Hisham AN, Lukman MR. Recurrent laryngeal nerve in thyroid surgery: a critical appraisal. ANZ J Surg 2002;72:887-9.

3. Gurleyik E, Gurleyik G. Incidence and Surgical Importance of Zuckerkandl's Tubercle of the Thyroid and Its Relations with Recurrent Laryngeal Nerve. ISRN Surg 2012;2012:450589.

4. Noussios G, Chatzis I, Konstantinidis S, et al. The Anatomical Relationship of Inferior Thyroid Artery and Recurrent Laryngeal Nerve: A Review of the Literature and Its Clinical Importance. J Clin Med Res 2020;12:640-6.

5. Cirocchi R, Arezzo A, D'Andrea V, et al. Intraoperative neuromonitoring versus visual nerve identification for prevention of recurrent laryngeal nerve injury in adults undergoing thyroid surgery. Cochrane Database Syst Rev 2019;1:CD012483.

Elizabeth Yi Wen Lee ${ }^{1} M B B S$,

Yijin Jereme Gan ${ }^{2}$ FAMS (Otorhinolaryngology),

Ming Yann $\underline{\operatorname{Lim}}^{2}$ FAMS (Otorhinolaryngology)

${ }^{1}$ Department of General Surgery, Changi General Hospital, Singapore

${ }^{2}$ Department of Otorhinolaryngology, Tan Tock Seng Hospital, Singapore

Correspondence: Dr Ming Yann Lim, Department of Otorhinolaryngology, Tan Tock Seng Hospital, 11 Jalan Tan Tock Seng, Singapore 308433.

Email: ming_yann_lim@ttsh.com.sg 